\title{
Bibliometrie
}

Isabella Peters* und Leon Ladewig

\section{Eine bibliometrische Analyse: Interdisziplinarität im Exzellenzcluster The Future Ocean}

https://doi.org/10.1515/iwp-2017-0063

Zusammenfassung: Der Artikel diskutiert Aspekte der Interdisziplinarität, um diese für eine bibliometrische Analyse des Exzellenzclusters „The Future Ocean“ zu operationalisieren. Das Exzellenzcluster wird als prototypische interdisziplinäre Forschungssituation angesehen, die im letzten Drittel des Förderzeitraums einer systematischen Bestandsaufnahme der interdisziplinären Kooperationen und des interdisziplinären Outputs bedarf. Als Indikatoren der Disziplinarität werden die institutionelle Herkunft der Autoren und Autorinnen über ihre Affiliation sowie die Zuordnung der Zeitschriften zu den Kategorien der Thomson ISI Essential Science Indicators (Stand: 2015), in denen die Artikel von Future Ocean erschienen sind, gewertet. Als Indizien für Interdisziplinarität werden Ko-Autorenrelationen und sog. Boundary Crossing betrachtet, das das Publizieren in fachfremden Disziplinen beschreibt. Die Ergebnisse der Analyse eignen sich als Steuerungsinstrument im Management von interdisziplinären Forschungssituationen, wie Future Ocean, und zeigen wichtige Handlungsoptionen zur Steigerung und Sicherstellung von Interdisziplinarität auf.

Deskriptoren: Interdisziplinär, Bibliometrie, Forschung, Meeresforschung, Exzellenzcluster, Boundary Crossing, Ko-Autoren-Analyse

\section{A Bibliometric Study: Interdisciplinarity of the Cluster of Excellence The Future Ocean}

\footnotetext{
Abstract: We discuss aspects of interdisciplinarity in order to operationalize them for a bibliometric analysis of the cluster of excellence "The Future Ocean". The cluster of excellence is a prototypical interdisciplinary research environment that requires a systematic evaluation of its inter-

*Kontaktperson: Prof. Dr.Isabella Peters,

ZBW - Leibniz-Informationszentrum Wirtschaft \& ChristianAlbrechts-Universität zu Kiel, Düsternbrooker Weg 120 , 24105 Kiel, E-Mail: i.peters@zbw.eu

Leon Ladewig, Technische Universität München, Institut für Informatik, Boltzmannstraße 3, 85748 Garching bei München, E-Mail: leon.ladewig@in.tum.de
}

disciplinary cooperation and output in the last third of its lifespan. We use the origin of authors via their affiliation and the background of journals, in which articles from Future Ocean have been published, via their allocation to the categories of the Thomson ISI Essential Science Indicators (2015) as indicators of disciplinarity. Interdisciplinarity will be operationalized by co-authorship-relations and so-called boundary crossing, which is defined as publishing in other disciplines than the home-discipline. The results of this study can be used by the management of the cluster since they reveal how interdisciplinary can be enhanced and secured.

Descriptors: Interdisciplinary, Bibliometrics, Research, Marine Research, Clusters of Excellence, Boundary Crossing, Co-Author Analysis

\section{Une analyse bibliométrique : l'interdisciplinarité dans le cluster d'excellence The Future Ocean}

Résumé: L'article traite des aspects de l'interdisciplinarité afin de les rendre opérationnels pour une analyse bibliométrique du cluster d'excellence The Future Ocean. Le cluster d'excellence peut être considéré comme un prototype de recherche interdisciplinaire qui nécessite un inventaire systématique de la production et des collaborations interdisciplinaires au cours du dernier tiers de la période de financement. En tant qu'indicateurs de discipline, les antécédents institutionnels des auteurs seront évalués sur leur affiliation, ainsi que l'attribution des revues aux catégories des Thomson ISI Essential Science Indicators (situation 2015), dans lesquels les articles de Future Ocean ont été publiés. Comme indice d'interdisciplinarité, on considère les relations entre co-auteurs et le «Boundary Crossing ", qui se réfère à la publication dans des disciplines tierces. Les résultats de l'analyse conviennent comme outil de contrôle dans la gestion des situations de recherche interdisciplinaire, comme Future Ocean, et montrent des options importantes pour accroître et assurer l'interdisciplinarité.

Descripteurs: Interdisciplinaire, Bibliométrie, Recherche, Recherche Marine, Cluster d'Excellence 


\section{Einleitung}

Spätestens seit das Research Excellence Framework (REF) 2014 in seiner Bewertung der britischen Forschungsleistung zeigte, dass Wissenschaft insbesondere dann eine Wirkung auf die Gesellschaft ausübt, wenn sie größtenteils interdisziplinär angelegt ist ${ }^{1}$, kann man sich der Bedeutung der Interdisziplinarität für Wissenschaft und Gesellschaft nicht mehr verschließen (Rylance, 2015; Allmendinger, 2015). Von interdisziplinärer Forschung erhofft man sich Fortschritt durch methodische und personelle Vielfalt (Umstätter, 1999) sowie eine höhere Anschlussfähigkeit an die Industrie, da sie sich eher praktischen Problemen und nicht der Grundlagenforschung widmet (Ledford, 2015).

Die meisten Initiativen der Forschungsförderer (wie EK oder DFG) legen daher verstärkt Wert auf Interdisziplinarität in geförderten Forschungsprojekten: „it funds interdisciplinary collaborations that seek genuine cross-fertilisation and deep synergies between the broadest range of advanced sciences [...] and cutting-edge engineering disciplines“" .

National fördern DFG und Wissenschaftsrat seit 2005 durch die Exzellenzinitiative von Bund und Ländern den Wissenschaftsstandort Deutschland ${ }^{3}$, wobei sie verschiedene Instrumente zum Ausbau der universitären Spitzenforschung finanzieren können. Gerade die Exzellenzcluster sollen eine interdisziplinäre Zusammensetzung und Ausrichtung sowie den Mehrwert durch Interdisziplinarität nachweisen ${ }^{4}$. Dies bestätigte auch der damalige DFGPräsident Kleiner in seinem Pressestatement zur zweiten Förderphase $2012^{5}$.

\subsection{Interdisziplinarität}

Obwohl das Konzept „Interdisziplinarität“ momentan stark beansprucht wird, ist seine Definition nicht unproblematisch (Fischer, 2011). Die Zeitschrift Nature widmete

\footnotetext{
1 https://www.hefce.ac.uk/media/HEFCE,2014/Content/Pubs/Indep endentresearch/2015/Analysis,of,REF,impact/Analysis_of_REF_impa ct.pdf [4.8.2017].

2 http://ec.europa.eu/research/participants/data/ref/h2020/wp/20 14_2015/main/h2020-wp1415-fet_en.pdf (S. 3) [4.8.2017].

3 http://www.dfg.de/foerderung/programme/exzellenzinitiative/all gemeine_informationen/index.html [4.8.2017].

4 Siehe http://www.dfg.de/formulare/exin305-67_70/exin305-67_70 .rtf und http://www.dfg.de/download/pdf/foerderung/programme/ exin/bewertungskriterien_exc_exin_120615.pdf [4.8.2017]. 5 http://www.dfg.de/download/pdf/dfg_im_profil/reden_stellung nahmen/2012/statement_kleiner_exin_120615.pdf [4.8.2017].
}

sich im Jahr 2015 der Interdisziplinarität in einem Schwerpunkthema ${ }^{6}$ und beschreibt interdisziplinäre Forschung als Synthese von disziplinären Elementen, die zu etwas komplett Neuem führt und eben nicht nur nebeneinander stattfindet. Ähnlich argumentiert Fischer (2011), wenn er die Systemlösung, als Gesamtpaket der Teillösungen, über die Fachprobleme stellt, aber die Notwendigkeit spezialisierter Forschung anerkennt. Parthey (2011, S. 13) konkretisiert und grenzt disziplinäre und interdisziplinäre Forschung voneinander ab: „Eine Forschungssituation ist disziplinär, wenn sich sowohl die in ihr formulierten Probleme als auch die in ihr verwendeten Methoden auf ein und denselben Bereich des theoretischen Wissens beziehen, und eine Forschungssituation ist interdisziplinär, wenn Problem und Methode der Forschung in verschiedenen Theorien formuliert bzw. begründet sind“. Derart interdisziplinäre Forschung führt manchmal dazu, dass neue Fächer in der disziplinären Landschaft installiert werden, wie Astrophysik, Biochemie, (Fischer, 2011).

Neben den definitorischen Unsicherheiten gibt es weitere Faktoren, die als Barrieren in der interdisziplinären Zusammenarbeit wahrgenommen werden. Interdisziplinäres Forschen wird unter Forschenden teilweise als Versagen in der Disziplin gewertet, was zudem die Karrieremöglichkeiten eingrenzt (Ledford, 2015). Es fehlen außerdem häufig die institutionalisierten Mittel wissenschaftlicher Kommunikation und Reputation, wie Gutachter und Zeitschriften, sodass weder die Bewertung noch die öffentliche Ausstellung interdisziplinärer Studien möglich ist. Nach Umstätter (1999) besteht in interdisziplinären Forschungssituationen eine gewisse Gefahr, Redundanz und Laienhaftigkeit in Publikationen und Studien zu erzeugen, weil die Fachexperten fehlen.

Neben der noch unzureichenden wissenschaftlichen Anerkennung ist gelebte Interdisziplinarität zudem anstrengend und erfordert den erhöhten Einsatz aller Betroffenen (Brown, Deletic, \& Wong, 2015). Da die Forschenden oft nicht die gleiche Terminologie verwenden, ist die Kommunikation ein Schlüsselfaktor für den Erfolg interdisziplinärer Projekte. Doch auch wenn die oben geforderte Synthese der Disziplinen gelingt, dauert die interdisziplinäre Forschung tendenziell länger, ist weniger effizient und damit teurer, was sowohl die Forschenden als auch die Förderer berücksichtigen müssen.

6 Nature, 535(7569), 17. Sep. 2015. 


\subsection{Bibliometrische Studien zur Interdisziplinarität}

Das Potential und die Ansätze bibliometrischer Studien zur Interdisziplinarität werden u.a. von Ball und Tunger (2005) beschrieben. Der Ansatz von Braun und Schubert (2003) arbeitet inhaltsbezogen und durchsucht Artikel in Fachzeitschriften nach den Begriffen „Interdisziplinarität“ und „Multidisziplinarität“ im Titel. So kann man den Diskurs zur Interdisziplinarität untersuchen, während ein zitatenanalytisches Vorgehen Hinweise auf die praktische Umsetzung von Interdisziplinarität (d.h. wenn Artikel aus anderen Disziplinen zitiert werden) liefert (Larivière \& Gingras, 2014). Die Grenzen des inhaltsbezogenen Ansatzes bestehen darin, dass hier allein die Sicht des Autors oder der Autorin bei der Bewertung der Interdisziplinarität berücksichtigt wird und nicht der tatsächliche (wahrgenommene) Impact des Artikels. Larivière und Gingras (2014) zeigen in einer umfassenden Studie zur Interdisziplinarität von Zeitschriftenartikeln aus den Jahren 1900 bis 2010, dass in den Artikeln aus den Sozialwissenschaften fünfmal häufiger die Zeichenfolge interdisziplin* erscheint, als in naturwissenschaftlichen Artikeln.

Für die Artikelebene zeigen mehrere Studien, dass der Großteil der interdisziplinäre Artikel kurzfristig eher weniger Zitationen als der Durchschnitt erzielen, wenn man aber einen längeren Zeitraum betrachtet, durchschnittlich mehr Zitationen akkumulieren als disziplinäre Artikel (Van Noorden, 2015; siehe auch Porter \& Rafols, 2009). Leahey, Beckman und Stanko (2017) stellen für interdisziplinär Publizierende einen „productivity penalty“ fest. Obwohl die Anzahl der Zitationen sich nicht signifikant von disziplinär Publizierenden unterscheidet, fällt die Anzahl der veröffentlichten Artikel für interdisziplinäre Autoren und Autorinnen geringer aus.

Die Menge der interdisziplinären Artikel steigt seit 1900 im Durchschnitt kontinuierlich an und ca. ein Drittel der untersuchten Artikel zitiert Quellen aus anderen Disziplinen (Larivière \& Gingras, 2014). Relativiert werden muss dieses Ergebnis, weil gleichzeitig auch eine Steigerung der Anzahl der Referenzen nachgewiesen werden kann. Der Anteil an interdisziplinären Referenzen seit 1930 ist daher nicht wesentlich gestiegen.

Ball und Tunger (2005) entwickeln ein Punktesystem zur Bewertung des Ausmaßes von Interdisziplinarität. Der Vorteil dieser Methode ist, dass, neben einer Übersicht des Einflusses des Artikels auf andere Disziplinen, die Anwendbarkeit und Übertragbarkeit der eigenen Ergebnisse evaluiert und z.B. zur Auseinandersetzung mit zukünftigen Anwendungsfeldern genutzt werden kann.
Damit werden auch die Grenzen von bibliometrischen Verfahren zur Untersuchung von Interdisziplinarität deutlich, die nur begrenzt Aussagen zu Ursache-Wirkung-Zusammenhängen machen können. Die hohe Korrelation zwischen Interdisziplinarität und Anzahl der Autoren und Autorinnen (Parthey, 1995) kann auch ein Effekt der zunehmenden Arbeitsteilung in der Wissenschaft sein, die verschiedene Expertisen erfordert, und muss nicht auf eine wirklich gelebte interdisziplinäre Forschungssituation zurückgehen. Der interdisziplinäre Charakter eines Forschenden entsteht oft auch daraus, dass Forschende im Laufe ihrer Karriere mehrfach Forschungsthemen und Fachgebiete wechseln (Umstätter, 1999).

\subsection{Fallbeispiel einer interdisziplinären Forschungssituation: Exzellenzcluster Future Ocean}

Als Beispiel einer interdisziplinären Forschungssituation widmet sich diese Arbeit dem Exzellenzcluster The Future Ocean (im Folgenden auch kurz als Cluster bezeichnet), das seit 2006 von der DFG gefördert wird. Kieler Forschungseinrichtungen, darunter die Christian-AlbrechtsUniversität zu Kiel (CAU) und das GEOMAR HelmholtzZentrum für Ozeanforschung Kiel (GEOMAR) sind daran beteiligt. Future Ocean befindet sich in der Tradition der außeruniversitären Forschungsinstitute, die besser als Universitäten die Möglichkeit bieten, „interdisziplinäre [...] Forschungssituationen zu schaffen und zu bearbeiten und zwar ungehindert durch die zwangsläufig disziplinären Lehrprofile an Universitäten“ (Parthey, 2011, S. 33). Das wissenschaftliche Erkenntnisinteresse von Future Ocean gilt der Veränderung der Ozeane; das Vorgehen erfolgt durch einen interdisziplinären Ansatz ${ }^{7}$. Dadurch ist der Cluster ein gutes Fallbeispiel für eine interdisziplinäre Forschungssituation mit einem disziplinenübergreifenden Problemfeld (Parthey, 2011), die es nun zu evaluieren gilt.

\subsection{Zielsetzung der Studie}

Für Parthey (2011) ist Interdisziplinarität in den Forschenden angelegt. Nach Fischer (2011) ist interdisziplinäres Denken zwar in den Genen von Forschungsverbünden der angewandten Wissenschaft per definitionem vorgesehen, muss aber gelebt werden, um erfolgreich zu sein: „Interdisziplinarität ist nicht bereits dann gewährleistet, wenn

7 http://www.futureocean.org/de/cluster/index.php [4.8.2017]. 
man Vertreter verschiedener Disziplinen oder Fächer in eine gemeinsame Arbeitsgruppe steckt“ (S. 55). Für die hier vorliegende Studie folgen wir daher dem Ansatz der DFG und untersuchen „Orte des Auftretens fachübergreifender Zusammenarbeit" ${ }^{* 8}$ auf ihre Interdisziplinarität. Orte fächerübergreifender Zusammenarbeit und von Wissenstransfer sind dann Ko-Autorenrelationen, da sie die Bereitschaft Forschender zur interdisziplinären Zusammenarbeit sowie erfolgreich stattgefundene Kollaboration widerspiegeln (Schummer, 2004). Diese Indikatoren nennt man combinations of knowledge und boundary crossing (Pierce, 1999), da sie das Vorkommen von fachfremden Autoren, Autorinnen oder Publikationen in einer Disziplin betrachten. Die Indikatoren der Disziplinarität sind die Zuordnung der Forschenden und ihrer Publikationen über eine Affiliation zu einer Disziplin (Ortega \& Antell, 2006) und die Zuordnung einer Publikation zu einer Journalkategorie der Thomson ISI Essential Science Indicators (ESI; Stand: 2015).

Wir wählen einen deskriptiven Ansatz, der eine Bestandsaufnahme durchführt, sich auf die Darstellung der vorhandenen Beziehungen des Clusters konzentriert und vor allem die Produkte interdisziplinärer Kooperation betrachtet. Die Reaktion auf diese, die man durch Zitationsanalysen oder Altmetrics zeigen könnte, wird nicht untersucht, um keine Leistungsbewertung der Clusteraktivitäten vorzunehmen.

Wir reihen uns damit in die Tradition bibliometrischer Studien zur Interdisziplinarität ein (u.a. Porter \& Rafols, 2009) und untersuchen, wo und in welchem Ausmaß Interdisziplinarität im Cluster sichtbar wird. Speziell werden folgende Forschungsfragen beantworten:

1. Welchen disziplinären Ursprung haben die Forschenden bzw. Publikationen des Clusters?

2. Wie lässt sich die Zusammenarbeit der Ko-Autoren und -Autorinnen strukturell beschreiben? Welche Autoren und Autorinnen tragen zentral zum interdisziplinären Wissensaustausch bei?

3. In welchen Disziplinen werden die im Cluster gewonnenen Erkenntnisse veröffentlicht?

Deskriptive Analysen eignen sich vor allem zur Information des Cluster-Managements und können gezielte Steuerungen vorbereiten. Zudem geben sie Hinweise darauf, an welchen Stellen Interdisziplinarität gut gelingt und an welchen Punkten Optimierungsbedarf besteht. Als Fallbeispiel wurde das Exzellenzcluster Future Ocean als

8 Siehe: http://www.dfg.de/dfg_profil/zahlen_fakten/evaluation_stu dien_monitoring/studien/bericht_interdisziplinaritaet/index.html [4.8.2017]. Siehe auch: Güdler (2013).
Prototyp einer interdisziplinären Forschungssituation gewählt, das sich zudem selbst eine erfolgreiche interdisziplinäre Zusammenarbeit zum Ziel gesetzt hat.

\section{Datenbasis und Methodik}

Die Untersuchung bezieht sich auf folgende zwei Analyseeinheiten: Autoren und Autorinnen sowie Publikationen. Bei den Forschenden aus dem Cluster wird ihre disziplinäre Zuordnung über ihre Affiliation erfasst und eine Ko-Autorenanalyse durchgeführt, um die interdisziplinäre Zusammenarbeit zu quantifizieren. Die Publikationen werden zudem auf ihren Publikationsort untersucht, also welcher Disziplin die veröffentlichende Zeitschrift zuzuordnen ist. Das Vorgehen folgt in großen Teilen der Studie von Schummer (2004).

\subsection{Datenbasis}

Den Ausgangspunkt für die bibliometrische Analyse bildet die auf der Website des Clusters zur Verfügung gestellte Publikationsdatenbank mit Stand von Mitte Februar 2015. Sie enthält 1.952 Publikationen aus dem Zeitraum Ende 2006 (Gründungsjahr des Clusters) bis Anfang 2015. Die Analyse beschränkt sich auf Artikel in Fachzeitschriften, Konferenzbeiträge und Artikel aus Sammelbänden.

Weiterhin wird nur ein Teil der 8.036 in der Publikationsdatenbank erfassten Autoren und Autorinnen untersucht, nämlich nur solche, die in der Mitgliederliste „Future Ocean Netzwerk" ${ }^{\text {"10 }}$ des Clusters aufgeführt werden. Für 56 der insgesamt 252 Mitglieder des Clusters kann keine Publikation nachgewiesen werden, da auch Mitglieder für andere Bereiche, beispielsweise Projektmanagement und Öffentlichkeitsarbeit, in der Cluster-Mitgliedsliste aufgeführt sind.

Nach obigen Einschränkungen bilden 1.605 Publikationen von 196 Autoren und Autorinnen (mit Mitgliedsstatus) die Grundlage für die folgenden Analysen. Tabelle 1 zeigt die Teilmengen der Datenbasis und in welchem Kapitel die Ergebnisse jeweils beschrieben werden. Die Analyse der Verbreitung von Forschungsinhalten über Disziplingrenzen hinweg wird später über die Zuordnung der Fachzeitschriften in den ESI (s. 2.4) erfolgen. Daher sind in

9 http://eprints.uni-kiel.de/view/divisions_all/FUTURE-OCEAN.date .html [4.8.2017].

$10 \mathrm{http}: / /$ www.futureocean.org/de/cluster/mitglieder/index.php (Stand: Februar 2015). 
Tabelle 1: Beschreibung des Datensatzes.

\begin{tabular}{|c|c|c|c|}
\hline Auswahl & $\begin{array}{r}\text { Ergebnispräsentation } \\
\text { in Kapitel }\end{array}$ & $\begin{array}{r}\text { Anzahl der } \\
\text { Publikationen }\end{array}$ & $\begin{array}{r}\text { Anzahl der } \\
\text { Autoren }\end{array}$ \\
\hline Gesamte Publikationsdatenbank (2006-2014) & & 1.952 & 8.036 \\
\hline $\begin{array}{l}\text { + Artikel, Konferenzbeiträge, Sammelbandbeiträge von Autoren und } \\
\text { Autorinnen mit Mitgliedsstatus }\end{array}$ & 3.1 & 1.605 & 196 \\
\hline++ mind. 2 Autoren und Autorinnen mit Mitgliedsstatus & 3.2 & 413 & 170 \\
\hline + Publikationen aus Journals aus ESI & 3.3 & 1.380 & 190 \\
\hline
\end{tabular}

Tabelle 1 auch Angaben zum Anteil der hier gefundenen Fachzeitschriften enthalten.

\subsection{Bestimmung der disziplinären Herkunft der Autoren und Autorinnen}

Für einen ersten Anhaltspunkt über die fachliche Zusammenarbeit der Autoren und Autorinnen ist interessant, in welchem Fachgebiet die jeweiligen Personen tätig sind. Aufschluss darüber gibt in den meisten Fällen bereits die Angabe „Forschungsschwerpunkt“ in der Mitgliederliste „Future Ocean Netzwerk“. Ist an dieser Stelle keine Information hinterlegt, kann über den Titel der Arbeitsgruppe bzw. Institution, in der die jeweiligen Forschenden tätig sind, auf ihren Forschungsschwerpunkt geschlossen werden. Letztlich geben auch Angaben zur Ausbildung im Lebenslauf des Mitglieds Hinweise auf die Disziplin. Die Zuordnung der Forschenden zu Disziplinen auf Basis der Affiliation und Forschungsschwerpunkte bietet den Vorteil, dass sie analog zu der konzeptionellen Struktur des Clusters erfolgt und damit die Disziplinen-Landschaft genauer widerspiegelt als z. B. über die artifizielle Zuordnung über andere Taxonomien (Schummer, 2004).

Allerdings ist aufgrund der Vielzahl von unterschiedlichen Fachbereichen, in die die Autoren und Autorinnen auf diese Weise eingeordnet werden können, eine Klassenbildung notwendig (Ortega \& Antell, 2006). Um eine größtmögliche Qualität in der Bestimmung der Disziplinzugehörigkeit $\mathrm{zu}$ erzielen, wurde manuell mit besonderer Berücksichtigung der disziplinären Cluster-Eigenheiten vorgegangen.
Tabelle 2: Kategorisierung der disziplinären Herkunft der Autoren und Autorinnen.

\begin{tabular}{|c|c|}
\hline Kategorie (Abkürzung) & $\begin{array}{l}\text { Erfasste Disziplinen nach Affiliation } \\
\text { (Auswahl) }\end{array}$ \\
\hline Biologie (Bio) & $\begin{array}{l}\text { Mikrobiologie, Zoologie, Botanik, } \\
\text { Physiologie, Molekularbiologie, } \\
\text { Humanernährung }\end{array}$ \\
\hline Biogeochemie (BGC) & Marine Biogeochemie \\
\hline Chemie (Ch) & $\begin{array}{l}\text { anorganische, organische, } \\
\text { physikalische Chemie, Pharmazie, } \\
\text { Toxikologie, funktionale } \\
\text { Nanomaterialien }\end{array}$ \\
\hline $\begin{array}{l}\text { Geisteswissenschaften } \\
\text { (Geist) }\end{array}$ & $\begin{array}{l}\text { VWL, Weltwirtschaft, Sozialwirtschaft, } \\
\text { Geschichte, Philosophie }\end{array}$ \\
\hline Geowissenschaften (Geo) & $\begin{array}{l}\text { Geologie, Sedimentologie, Geographie, } \\
\text { Küstengeowissenschaften, weitere } \\
\text { Geowissenschaften }\end{array}$ \\
\hline Geophysik (GeoPh) & $\begin{array}{l}\text { Geodynamik, marine Geodynamik, } \\
\text { magmatische und hydrothermale } \\
\text { Systeme, Geosysteme, } \\
\text { Altersbestimmung, Isotopenforschung }\end{array}$ \\
\hline Jura (Jur) & Seerecht \\
\hline Mathematik (Ma) & $\begin{array}{l}\text { Informatik, Informations- und } \\
\text { Codierungstheorie, experimentelle } \\
\text { Numerik }\end{array}$ \\
\hline Medizin (Med) & Marine Medizin \\
\hline Meteorologie (Meteo) & Marine Meteorologie \\
\hline Ökologie (Öko) & $\begin{array}{l}\text { Marine Ökologie, Evolutionsökologie, } \\
\text { experimentelle Ökologie, } \\
\text { Ökosystemforschung }\end{array}$ \\
\hline Ozeanografie (Ozean) & $\begin{array}{l}\text { physikalische, chemische, biologische } \\
\text { Ozeanografie, Paläoozeanografie }\end{array}$ \\
\hline
\end{tabular}

$\mathrm{Ab}$ einer Mitgliederzahl von fünf Autoren und Autorinnen wurde eine Kategorie für den entsprechenden Fachbereich angelegt. Für Autoren und Autorinnen mit eher spezifischem Fachbereich, der weniger als fünfmal vertreten war, wurde eine Kategorie mit einem Oberbegriff (etwa Biologie) angelegt, der den größtmöglichen thematischen Nenner widerspiegelt. Dies führt dazu, dass die Kategorie 
Geophysik neben Geowissenschaften existiert, obwohl man inhaltlich die Geophysik als eine Geowissenschaft charakterisieren würde. In Geowissenschaften sind alle übrigen Geowissenschaften untergebracht, die nicht Teil der Geophysik sind. Zudem heben sich einige Fachbereiche (z.B. Jura) so stark vom naturwissenschaftlichen Feld ab oder sind auf andere Weise speziell, dass sie trotz Unterschreitung der Mindestanzahl der Autoren und Autorinnen eine eigene Kategorie bilden. Jeder Autor und jede Autorin wurden nur einer Kategorie zugeordnet.

Die Klassenbildung, Benennung der Kategorien und Zuordnung der Autoren und Autorinnen lässt Raum für Optimierung (z.B. hätte die Kategorie Geisteswissenschaften auch Sozialwissenschaften genannt werden können). Genauso wie Ortega und Antell (2006) arbeiten wir mit einem anspruchsvollen Verständnis von „Interdisziplinarität“, von ihnen bezeichnet als „cross-disciplinary“ (S. 447), um Verbindungen zwischen weiter voneinander entfernten Disziplinen aufzuzeigen. Aufgrund der Mehrheit der naturwissenschaftlich Forschenden im Cluster wurden aber gerade diese Disziplinen doch voneinander getrennt, um hier spezifischere Einblicke in die interdisziplinäre Forschung zu ermöglichen und für ein Fallbeispiel einen Überblick über die interdisziplinäre Zusammenarbeit zu geben. Tabelle 2 zeigt die gewählten Kategorien sowie eine Auswahl der darin enthaltenen Disziplinen.

\subsection{Ko-Autorennetzwerke}

Die Ko-Autorenanalyse ist ein pragmatisches Mittel in bibliometrischen Studien (Havemann \& Scharnhorst, 2010), um die Interdisziplinarität von Forschungssituationen zu ermitteln. Die gemeinsame Verantwortung eines Zeitschriftenartikels setzt die Bekanntschaft der Autoren oder Autorinnen voraus. Außerdem lässt sich vom disziplinspezifischen Background der Autoren und Autorinnen zumindest das Potential für die interdisziplinäre Bearbeitung disziplinübergreifender Probleme (nach Parthey, 2011) ermitteln. Ko-Autorenanalysen bieten den Vorteil, dass sie die Forschungspraxis widerspiegeln und nicht nur die Informationsbeschaffung und -distribution von und durch die Autoren und Autorinnen reflektieren. Sie zeigen außerdem eine erfolgreiche Kooperation zwischen Disziplinen an (Schummer, 2004).

Mehrere Autoren und Autorinnen, die gemeinsam als Verfassende einer Publikation angegeben sind, stehen paarweise in Ko-Autorenrelation zu einander. Die Analyse resultiert häufig in Ko-Autorennetzwerken, die entweder ungewichtet oder gewichtet sein können. Im ersten Fall wird lediglich nach dem (Nicht-)Vorliegen einer Ko-Autor- schaft gefragt, im letzteren Fall wird die Anzahl der gemeinsam erstellten Artikel zur Ermittlung der Verbindungsstärke zwischen den Autoren und Autorinnen genutzt. Letztere bestimmt damit die Position der Verfassenden im Netzwerk. Ko-Autorennetzwerke lassen sich so mit Mitteln der Sozialen Netzwerk-Analyse untersuchen und beschreiben. In früheren Studien konnte gezeigt werden, dass sich häufig mehr als 80 Prozent der Autoren und Autorinnen aus einem Fach im sog. main component des Netzwerks befinden, also intensive Ko-Autorenbeziehungen zu anderen Forschenden pflegen (Newman, 2001).

Für die übersichtliche Darstellung der Ko-Autorenrelationen bietet sich ein ungerichteter Graph mit gewichteten Kanten an. Darin entsprechen Knoten den Autoren und Autorinnen und Kantengewichte dem Ausprägungsgrad ihrer Ko-Autorenrelation (Ohly, 2003). Durch eine Einfärbung der Knoten können weitere Informationen - etwa die Fachbereiche der jeweiligen Autoren und Autorinnen - im Graph untergebracht werden. Zur Analyse und Visualisierung der Autorennetzwerke diente in dieser Studie die Open-Source-Software Gephi ${ }^{11}$. Für die Auswertung der KoAutorenbeziehung wurde der in Gephi implementierte Modularitätsalgorithmus von Blondel, Guillaume, Lambiotte und Lefebvre (2008) genutzt, wobei eine Auflösung von 1.0 und Randomisierung als Einstellungen gewählt wurde.

In dieser Studie zählen Beiträge durch Einzelautorschaft nicht, da sie keinen Einfluss auf das Ko-Autorennetzwerk des Clusters haben ${ }^{12}$. Das bedeutet, dass nur Publikationen betrachtet werden, die von mindestens zwei Autoren und Autorinnen aus dem Cluster verfasst worden sind. Daher fließen in die Analyse 413 Publikationen ein, was in etwa 21 Prozent der Gesamtmenge erhältlicher Publikationen aus dem Cluster (s. Tab. 1) entspricht. Hieraus könnte man zunächst auf eine Mehrheit von Publikationen durch Einzelautorschaft schließen, was aber relativiert werden muss: Bei der Auswertung aller jeweils angegebenen Autoren und Autorinnen wird vielmehr klar, dass Einzelautorschaft die Ausnahme darstellt. An jeder der 1.605 Publikationen sind durchschnittlich vier Autoren und Autorinnen beteiligt. Dieses deutliche Indiz für Mehrautorenpublikationen deckt sich mit der Studie von Littke (2009), die in den Geowissenschaften (s. 3.1) Mehrautorschaft als Regelfall aufgezeigt hat.

Einzelne Artikel mit ausgeprägter Mehrautorschaft (z.B. mit 63 Autoren und Autorinnen) sind häufig den

11 https://gephi.org [4.8.2017].

12 Einzelautorschaft bedeutet hier, dass nur ein Autor oder eine Autorin aus dem Cluster bei der Erstellung der Publikationen involviert war. Die Publikation kann aber durchaus mehrere Autoren und Autorinnen haben. 
Medizinern im Cluster zuzuschreiben. Noth und Rose (2009) beobachten derart lange Autorenlisten insbesondere bei großangelegten klinischen Studien und erklären dies mit dem breiten wissenschaftlichen Spektrum, das zur Durchführung einer solchen Studie erforderlich ist.

\subsection{Interdisziplinäre Verbreitung von Forschungsergebnissen}

Weitere Indizien für die Interdisziplinarität von Forschung sind der thematische Hintergrund des Publikationsortes (z. B. Fachzeitschrift oder Konferenz; Pierce, 1999) und seine Beziehung zu Thema und der disziplinären Herkunft der Autoren und Autorinnen. Oft richtet sich der Publikationsort an eine Zielgruppe, deren Fachbereich bestimmbar ist. Parthey (2011) nennt Zeitschriften daher auch „Spezialbibliotheken“, die das Wissen einer Disziplin erfassen. So lässt sich durch Zeitschriften nachzeichnen, wohin die Forschungsergebnisse distribuiert und in welchen Disziplinen sie rezipiert werden. Nach Pierce (1999) ist es vorteilhaft, dass das Boundary Crossing eine direkte Form des interdisziplinären Wissenstransfers ist, das Autoren und Autorinnen erlaubt „to communicate directly across disciplinary lines, instead of depending upon researchers in other disciplines to interpret and reference their work" (S. 271).

In der zur Verfügung stehenden Publikationsdatenbank von Future Ocean machen Artikel über 90 Prozent der erfassten Dokumenttypen aus. Übrig bleiben für die Analyse 1.453 Artikel, die in 631 verschiedenen Fachzeitschriften erschienen sind (und für die das Mehrautorenkriterium aus Clustermitgliedern aufgehoben wurde).

Um die Zeitschriften grob in Disziplinen zu unterteilen, wird auf die in der Datenbank Thomson ISI Essential Science Indicators $(\mathrm{ESI})^{13}$ verwendeten Kategorien zurückgegriffen. Dort wird jeder Zeitschrift eine von 22 Disziplinen zugeordnet, zum Beispiel der Zeitschrift Progress in Oceanography die Kategorie Geosciences. Aus der ClusterPublikationsdatenbank können 1.380 Artikel extrahiert werden, die in Journals erschienen sind, welche im ESI indexiert worden sind.

Im Gegensatz zu den Subject Categories im Web of Science des Institute for Scientific Information (ISI) wird hier jede Zeitschrift nur einer Kategorie zugeordnet, sodass multiple Kategorien pro Zeitschrift vermieden werden. Über die Kategorisierung der Zeitschriften wird ermittelt, wie oft Autoren und Autorinnen bestimmter Fachbereiche in Zeitschriften der zugehörigen oder einer anderen Kategorie veröffentlicht haben. Publikationen mehrerer Autoren und Autorinnen werden dabei mehrfach gezählt: Hat ein Biologe gemeinsam mit einer Ozeanografin einen Artikel in Progress in Oceanography veröffentlicht, wird sowohl der Disziplin Biologie als auch der Ozeanographie je eine Publikation in einer Zeitschrift der Kategorie Geosciences zugerechnet. Insgesamt lassen sich so 1.864 Zuordnungen für 1.380 Publikationen herstellen (s. Tab. 6).

\section{Ergebnisse}

Es folgen die Ergebnisse der Auswertung der disziplinären Herkunft der Autoren und Autorinnen, der Interdisziplinarität der Forschungskooperationen und der interdisziplinären Verbreitung der wissenschaftlichen Ergebnisse über Zeitschriften.

\subsection{Disziplinäre Herkunft der Autoren und Autorinnen}

In Abbildung 1 ist die Verteilung der disziplinären Herkunft der insgesamt 196 Autoren und Autorinnen dargestellt. Die meisten Autoren und Autorinnen sind den Fachbereichen Geophysik und Ökologie zuzuordnen. Auch das Gebiet der Geowissenschaften mit 26 Mitgliedern (übrige Geowissenschaften, außer Geophysik) gehört zu den am häufigsten vertretenen Disziplinen im Cluster. In etwa gleich viele Autoren und Autorinnen sind in der Chemie sowie in der Biogeochemie beheimatet. Die Ozeanografie und Meteorologie stellen einen wesentlichen Teil der Clustermitglieder, genauso wie die Mathematik, die auch Mitglieder aus der Informatik der CAU enthält.

Der Bereich der Geisteswissenschaften ist ebenfalls gut repräsentiert und unterscheidet sich von den anderen Kategorien durch die beteiligten Institutionen: Mit dem Institut für Weltwirtschaft und der Muthesius Kunsthochschule sind hier Institutionen involviert, die ausschließlich in diesem Bereich wirken. Einen kleinen und (von der Disziplin her) eher peripheren Fachbereich im Cluster bilden vier Forschende aus der Medizin, die sich mit dem Forschungsschwerpunkt Marine Medizin beschäftigen. Der Fachbereich Jura wird allein durch Matz-Lück mit ihrer Professur für Seerecht vertreten.

13 http://ipscience-help.thomsonreuters.com/incitesLiveESI/ESIGro up/overviewESI/esiJournalsList.html (Stand: 2015). 


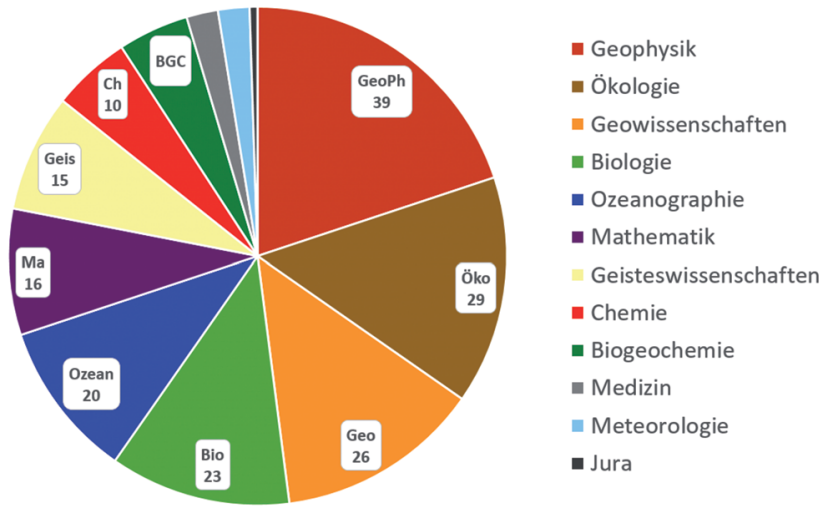

Abbildung 1: Disziplinäre Herkunft der Autoren und Autorinnen nach institutioneller Zugehörigkeit ( $n=196)$. Erläuterung zu Abkürzungen siehe auch Tabelle 2.

\subsection{Analyse der Ko-Autorschaft}

Dieses Kapitel beschreibt die Ko-Autorenbeziehungen und geht insbesondere auf die dadurch sichtbar werdenden Communities und zentralen Autoren und Autorinnen im Netzwerk ein. Abbildung 2 zeigt den vollständigen Graphen des Ko-Autorennetzwerks, entsprechend der in Abschnitt 2.3 eingeführten Methodik.

\subsubsection{Ko-Autorennetzwerke und Communities}

Communities im Ko-Autorennetzwerk deuten auf häufige Zusammenarbeiten und gemeinsame Publikationen einzelner Mitglieder hin. Für unseren Datensatz können auf Basis der 413 Publikationen von 170 Autoren 17 Communities ausfindig gemacht werden (mit Größen von 3 bis 21 Mitgliedern), die auch im Layout des Graphen aus Abbildung 2 berücksichtigt wurden. Autoren und Autorinnen, die sich in einer Community befinden, werden in räumlicher Nähe dargestellt.

Die meisten der 17 Communities sind miteinander verbunden, was die enge Kooperation unter den Clustermitgliedern verdeutlicht und schon von Newman (2001) für andere Ko-Autorenbeziehungen festgestellt wurde. Besonders markant ist die enge Zusammenarbeit zwischen Schreiber (Med) und Rosenstiel (Bio). Gemeinsam haben sie an 48 Publikationen mitgewirkt, was weit über dem Durchschnitt des Clusters liegt. Lediglich vier Communities sind vom Rest isoliert. An den isolierten Communities fällt ihre geringe Größe auf: Sie bestehen meist aus zwei (R. Schulz / Maser, Peterson / Klepper) bzw. drei Autoren und Autorinnen (C. Schulz / Samuel-Fitwi / Schröder, Koch / Köser / Jordt).
Betrachtet man nun zusätzlich, zu welchem Fachbereich die jeweiligen Mitglieder der Communities gehören, gibt es einerseits Gruppen, die deutlich von bestimmten Fachbereichen (nach der Zuordnung in Tabelle 2) geprägt sind: Beispielsweise besteht die Community um Kläschen hauptsächlich aus Forschenden der Geophysik (11 von 18 Mitgliedern) und Geowissenschaft (5 von 18 Mitgliedern). Kooperiert wird mit Harms aus der Biologie und Snower aus den Geisteswissenschaften (VWL). Diese Community besteht damit aus vier verschiedenen Disziplinen, die interdisziplinär publizieren, aber thematisch stark durch die Geophysik/Geowissenschaft beeinflusst werden.

Andere Communities spiegeln die disziplinäre Fokussierung und Zusammenarbeit noch stärker wider, z.B. die Gruppe um Schreiber. Es sind nur zwei Disziplinen vertreten, nämlich Biologie ( 5 von 8 Mitgliedern) sowie Medizin (3 von 8 Mitgliedern). Drei der insgesamt vier Mediziner aus dem Cluster lassen sich dieser Community zuordnen. Der vierte Mediziner (Schröder) gehört einer der isolierten Communities an.

Andererseits finden sich auch Communities, die durch ihre fachliche Zusammensetzung eine starke interdisziplinäre Herangehensweise vermuten lassen und ein breites thematisches Spektrum mit ihren Publikationen abdecken. In der vierzehnköpfigen Gruppe um Dierking (oben rechts in Abbildung 2) sind viele Forschende aus den Geisteswissenschaften (6 Mitglieder), aber auch aus der Ökologie (5 Mitglieder) sowie je ein Mitglied aus Geowissenschaften, Ozeanografie und Jura vertreten.

\subsubsection{Zentrale Autoren und Autorinnen}

Zentrale Forschende aus dem Ko-Autorennetzwerk (Abb. 2) zeichnen sich dadurch aus, dass sie Verbindungen zwischen den Mitgliedern des Clusters und den Communities herstellen und als Vermittler für den Wissensaustausch innerhalb des Exzellenzclusters Future Ocean sorgen. Netzwerktheoretisch werden solche Knoten durch die Betweenness Centrality bestimmt, die den Anteil der kürzesten Wege zwischen zwei Autorinnen angibt, auf denen ein Knoten liegt (Wasserman \& Faust, 1994).

In Tabelle 3 ist die Betweenness Centrality (normiert, d.h. als prozentualer Anteil an der Gesamtzentralität) der zehn Autoren und Autorinnen mit den höchsten Werten angegeben. Dass die aufgeführten Autoren und Autorinnen eine zentrale Position im Ko-Autorennetzwerk einnehmen, stimmt in den meisten Fällen mit der Einschätzung überein, die sich durch bloßes Betrachten von Abbildung 2 einstellt. Teilt man den Graphen vertikal etwa mittig durch, wird deutlich, dass viele der geschnittenen Ko-Autorenverbin- 


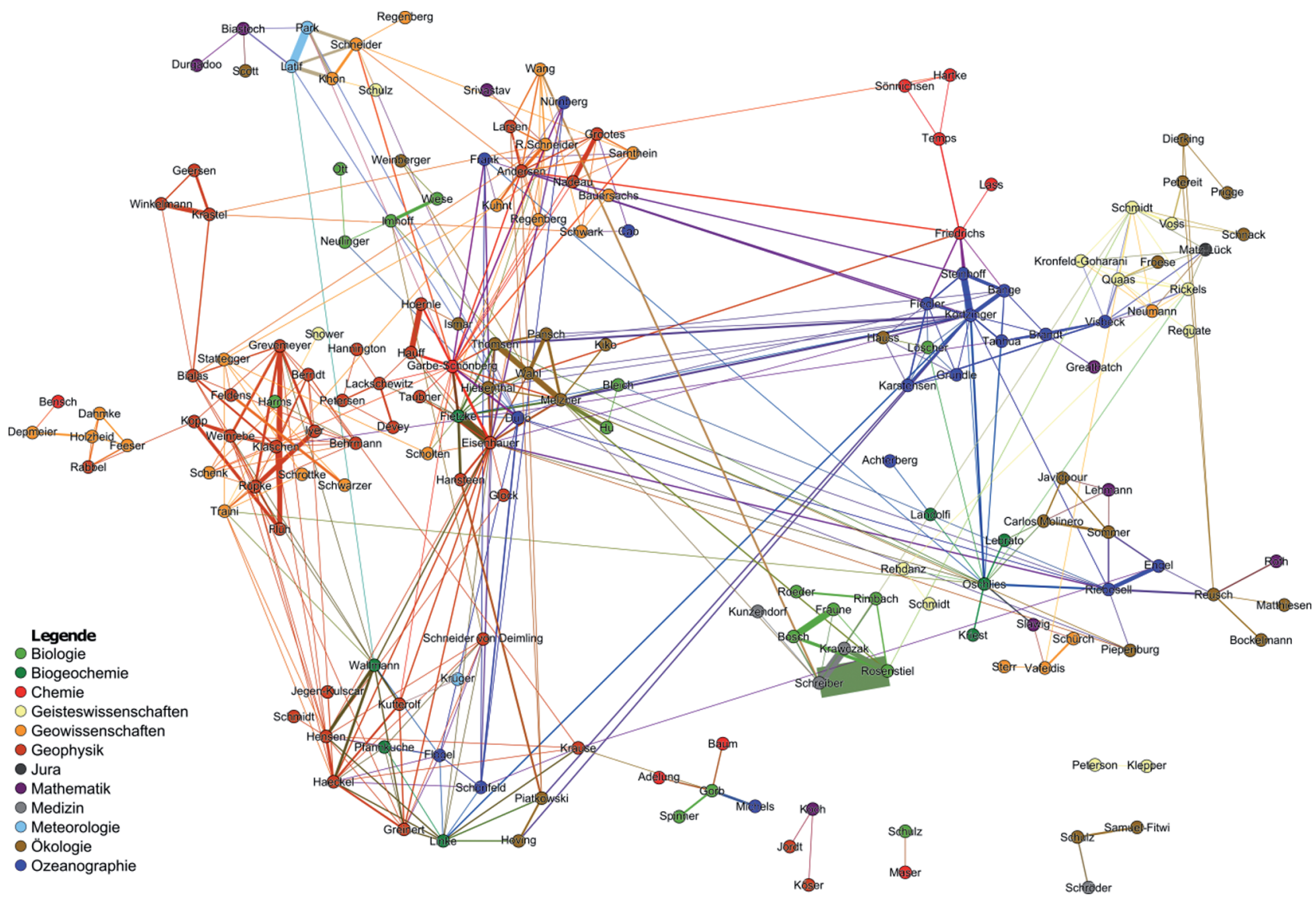

Abbildung 2: Ko-Autorennetzwerk ( $n=196)$. Autoren und Autorinnen sind entsprechend ihrem Fachbereich eingefärbt.

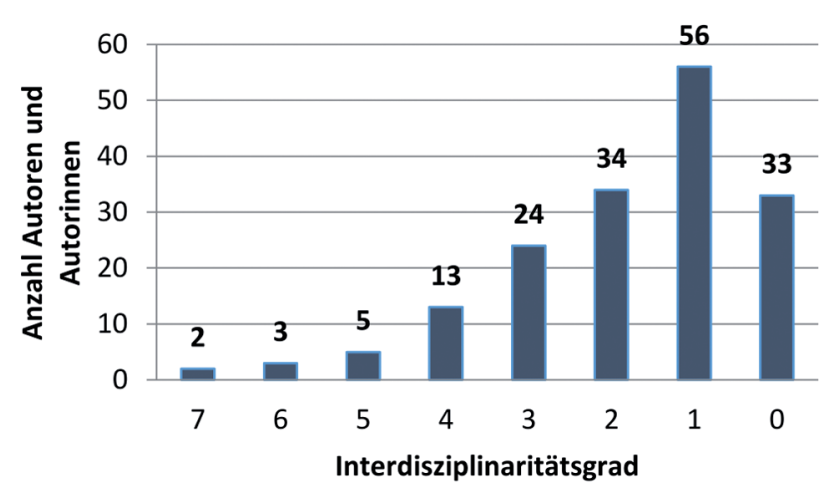

Abbildung 3: Verteilung der Interdisziplinaritätsgrade ( $n=170$ Autoren und Autorinnen).

dungen in der rechten Hälfte mit Körtzinger, Oschlies und Riebesell verbunden sind. Analog sind dies in der linken Hälfte vor allem Eisenhauer, Garbe-Schönberg und Melzner (gehören sogar zu einer einzigen Community). Die sechs genannten Autoren nehmen die sechs ersten Ränge in der Reihenfolge nach Betweeness Centrality ein.

Neben der Betweeness Centrality ist auch die Anzahl der interdisziplinären Verbindungen dieser Autoren und
Autorinnen interessant, weil dies Auskunft über die Entstehung ihrer zentralen Rolle gibt. Dazu wurde für jeden Autor und jede Autorin erfasst, mit wie vielen weiteren Disziplinen er oder sie bereits publiziert hat, wobei hier ein mengentheoretischer Ansatz für die Interdisziplinaritätsmetrik gewählt wurde (s. Abb. 3). Der höchste Wert beträgt sieben, d.h. solche Autoren und Autorinnen haben Ko-Autorschaften mit sieben anderen Disziplinen außerhalb der eigenen. Dies ist bei zwei Autoren (Dullo und Oschlies) der Fall. Mehr als zwei Drittel der Autoren und Autorinnen (67\%) publizieren mit einer, zwei oder drei weiteren Disziplinen. Über keine interdisziplinären Verbindungen verfügen 33 Autoren und Autorinnen, also knapp 20 Prozent der 170 Autoren und Autorinnen dieser Analyse. Der durchschnittliche Interdisziplinaritätsgrad der Forschenden des Future Ocean Clusters liegt bei 1,79 .

Von den fünf Autoren mit einem Interdisziplinaritätsgrad von $>=6$ sind drei unter den Top-15 der BetweenessCentrality-Rangliste. Ebenso gehören aber auch Autoren und Autorinnen mit niedrigerem Interdisziplinaritätsgrad $\mathrm{zu}$ dieser Rangliste, insbesondere Kopp mit einem Grad von 0. Dies zeigt, dass Autoren und Autorinnen innerhalb 
Tabelle 3: Betweeness Centrality und Interdisziplinarität von den Top-15-Autoren und -Autorinnen (sortiert nach Betweeness Centrality).

\begin{tabular}{lllr}
\hline Autor/Autorin & Fachbereich & $\begin{array}{r}\text { Betweeness } \\
\text { Centrality (normiert) }\end{array}$ & $\begin{array}{r}\text { Interdisziplinaritätsgrad } \\
\text { des Autors/der Autorin }\end{array}$ \\
\hline Oschlies, Andreas & BGC & 0,173 & 7 \\
\hline Eisenhauer, Anton & GeoPh & 0,161 & 5 \\
\hline Melzner, Frank & Öko & 0,123 & 6 \\
\hline Garbe-Schönberg, Dieter & Ch & 0,121 & 6 \\
\hline Körtzinger, Arne & Ozean & 0,104 & 5 \\
\hline Riebesell, Ulf & Ozean & 0,089 & 4 \\
\hline Schneider, Ralph R. & Geo & 0,082 & 5 \\
\hline Traini, Camille & Geo & 0,069 & 3 \\
\hline Krause, Stefan & GeoPh & 0,065 & 3 \\
\hline Kopp, Heidrun & GeoPh & 0,065 & 0 \\
\hline Dullo, Christian & Ozean & 0,060 & 7 \\
\hline Schmidt, Jörn & Geist & 0,046 & 6 \\
\hline Fietzke, Jan & BGC & 0,032 & 5 \\
\hline Schneider, Birgit & Geo & 0,030 & 5 \\
\hline Wahl, Martin & Öko & 0,029 & 5 \\
\hline
\end{tabular}

ihrer disziplinären Communities zentrale Positionen einnehmen können, jedoch nicht unbedingt für den interdisziplinären Austausch sorgen müssen (wie auch der positive, aber niedrige Korrelationswert nach Pearson $r=0,287$ zeigt). Fest steht jedoch, dass der Interdisziplinaritätsgrad unter den Top-15-Autoren und -Autorinnen nach Betweeness-Centrality deutlich über dem Durchschnitt aller Autoren und Autorinnen (1,79) liegt, nämlich bei 4,4.

\subsubsection{Zusammenarbeit der einzelnen Disziplinen}

Neben der Untersuchung der Interdisziplinarität von autor-basierten Netzwerken ist es auch von Interesse, ob bestimmte Disziplinen eher dazu neigen, interdisziplinär zu arbeiten, ob bestimmte Kooperationen gehäuft auftreten, oder ob die Zusammenarbeit eher ad-hoc stattfindet und von weiteren Faktoren abhängt (z. B. die Persönlichkeit der Forschenden).

Dazu wurden die Angaben zu den Ko-Autorschaften auf Disziplin-Ebene aggregiert (Publikationen mit mehreren Autoren und Autorinnen wurden mehrfach gezählt; s. Tab. 4). Von den 428 Ko-Autorenbeziehungen bestehen 179 nur innerhalb der Disziplin, 249 Ko-Autorenbeziehungen sind interdisziplinär. Zur besseren Veranschaulichung dient der Graph aus Abbildung 4, der nur solche Verbindungen enthält, die in mindestens fünf Publikationen vorkommen (daher sind Jura und Meteorologie nicht vertreten), und in dem reflexive, d.h. zwischen den gleichen
Disziplinen verlaufende, Verbindungen (Diagonaleinträge in Tabelle 4) ausgeschlossen wurden.

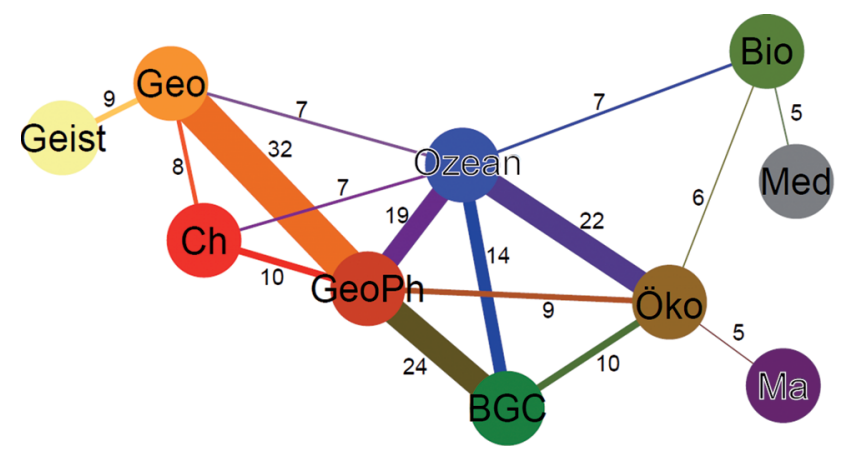

Abbildung 4: Zusammenarbeit der Disziplinen auf Basis von mehr als fünf gemeinsamen Publikationen.

Die der Biogeochemie inhärente Interdisziplinarität spiegelt sich auch in den Ko-Autorenrelationen wider, wobei die meisten Verbindungen zu Geophysik, Ozeanografie und Ökologie bestehen. Die Geophysik hat ausgeprägte Verbindungen in die Bereiche Geowissenschaften, Ökologie, Biogeochemie und Ozeanografie, was vor allem auf die spezifische Schwerpunktsetzung des Exzellenzclusters zurückzuführen ist. Unter den wenigen Fachbereichen, die mit der Medizin verbunden sind, zeigt die Biologie die am meisten ausgeprägte Verbindung. Ausgehend von der Ozeanografie verlaufen Ko-Autorenverbindungen in nahezu alle Disziplinen, am häufigsten in Richtung der Geophy- 
Tabelle 4: Zusammenarbeit der Disziplinen auf Basis gemeinsamer Publikationen ( $n=428$ Ko-Autorenbeziehungen).

\begin{tabular}{|c|c|c|c|c|c|c|c|c|c|c|c|c|}
\hline & BGC & Bio & Ch & Geist & Geo & GeoPh & Jur & $\mathrm{Ma}$ & Med & Meteo & Ozean & Öko \\
\hline BGC & 4 & & & & & & & & & & & \\
\hline Bio & 1 & 10 & & & & & & & & & & \\
\hline $\mathrm{Ch}$ & 1 & 3 & 5 & & & & & & & & & \\
\hline Geist & 2 & 1 & 0 & 11 & & & & & & & & \\
\hline Geo & 4 & 0 & 8 & 9 & 22 & & & & & & & \\
\hline GeoPh & 24 & 3 & 10 & 0 & 32 & 74 & & & & & & \\
\hline Jur & 0 & 0 & 0 & 4 & 1 & 0 & 0 & & & & & \\
\hline Ma & 1 & 0 & 0 & 0 & 3 & 2 & 0 & 1 & & & & \\
\hline Med & 0 & 5 & 0 & 1 & 1 & 0 & 0 & 0 & 2 & & & \\
\hline Meteo & 1 & 0 & 1 & 0 & 4 & 1 & 0 & 2 & 0 & 1 & & \\
\hline Ozean & 14 & 7 & 7 & 4 & 7 & 19 & 1 & 1 & 0 & 2 & 26 & \\
\hline Öko & 10 & 6 & 4 & 4 & 0 & 9 & 0 & 5 & 2 & 0 & 22 & 23 \\
\hline
\end{tabular}

sik, Ökologie und Biogeochemie. Allein mit dem Fachbereich Medizin ist keine Ko-Autorschaft festzustellen. Obwohl es auch eine hohe Anzahl an Ko-Autorschaften innerhalb der Ozeanografie gibt (26 von 58 Kooperationen), sprechen das breite Spektrum der Kooperationen und die allgemein hohe Zahl an Ko-Autorschaften deutlich für ein disziplinübergreifendes Veröffentlichungsverhalten.

Die Geisteswissenschaften erscheinen in Abbildung 4 fast isoliert, da sie nur bei den Geowissenschaften auf fünf oder mehr Publikationen kommen. Weniger häufige Kooperationen bestehen $\mathrm{zu}$ verschiedenen Naturwissenschaften (BGC, Bio, Ozean, Öko) und Jura. Tabelle 4 ist weiterhin zu entnehmen, dass verhältnismäßig viele KoAutorenbeziehungen innerhalb des geisteswissenschaftlichen Bereichs verlaufen (11 von 35 Kooperationen). Aus dem Gebiet der Mathematik verläuft in Abbildung 4 nur eine Verbindung zu der Ökologie, weniger Kooperationen bestehen zu unterschiedlichen Fachgebieten. Forschungsergebnisse aus der Mathematik dienen anderen Fachbereichen vorwiegend als Grundlage, indem beispielsweise ein mathematisches Modell des globalen Klimasystems entwickelt wird. Modellierung könnte dann als eine Art Hilfswissenschaft im Cluster angesehen werden.

\subsection{Interdisziplinäre Verbreitung von Forschungsergebnissen}

Nach der Analyse der Ko-Autorenbeziehungen folgt nun die Auswertung der Publikationsorte, in denen die Ergebnisse dieser Kooperationen präsentiert werden. Eine Betrachtung der thematischen Kategorien, die Fachzeitschriften im ESI zugeordnet werden, gibt Aufschluss über die Verbreitungsweise interdisziplinärer Ergebnisse und damit indirekt auch über das Potential über-disziplinärer Rezeption und Reputation, die die Forschenden erlangen können. Man erfährt auch, welche Zeitschriften vom Cluster adressiert werden und welche Journale interdisziplinäre Ergebnisse besonders häufig aufnehmen. Tabelle 5 zeigt, wie oft Forschende bestimmter Fachbereiche in Zeitschriften einzelner Kategorien veröffentlichen (die Artikel wurden den Kategorien auf Basis der Ko-Autorenrelationen mehrfach zugeordnet). Als erstes ist festzuhalten, dass in jeder der insgesamt 22 ESI-Kategorien (s. 2.4) mindestens ein Artikel aus dem Cluster erschienen ist. Dies spricht bereits für eine inhaltliche Bandbreite bei den adressierten Zeitschriften.

Die im Cluster am häufigsten vertretenen Journal-Kategorien sind Geosciences (599 Artikel), Environment / Ecology (206 Artikel) sowie Plant \& Animal Science (176 Artikel). Am wenigsten Artikel erschienen dagegen in den Kategorien Space Science (ein Artikel aus der Biologie), Psychiatry/Psychology (vier Artikel aus den Geisteswissenschaften) und Neuroscience \& Behavior (sieben Artikel aus verschiedenen Disziplinen). Die Kategorie Multidisciplinary (mit Zeitschriften wie Science und Nature) steht mit 108 Artikeln auf dem siebten Rang. Die einzige Publikation aus dem Bereich Jura erschien in der Zeitschrift Marine Policy. Diese ist der Zeitschriftenkategorie Social Sciences zugeordnet.

Die Zahl der Kategorien, in denen die einzelnen Fachbereiche veröffentlichen, variiert stark: Geophysik und Meteorologie sind Beispiele dafür, dass eine Kategorie (Geosciences) dominiert. Ein breites Spektrum an Kategorien deckt der Fachbereich Biologie ab. Von den 22 JournalKategorien haben lediglich zwei keine Veröffentlichung 
Tabelle 5: Anzahl der Publikationen aus verschiedenen Disziplinen (mehrfach zugewiesen für jeden Autor und für jede Autorin; horizontal) in Zeitschriften jeweiliger Kategorie (vertikal).

\begin{tabular}{|c|c|c|c|c|c|c|c|c|c|c|c|c|c|}
\hline & BGC & Bio & $\mathrm{Ch}$ & Geist & Geo & GeoPh & Jur & Ma & Med & Meteo & Ozean & öko & Total \\
\hline AGRICULTURAL SCIENCES & 0 & 21 & 0 & 0 & 0 & 3 & 0 & 0 & 2 & 0 & 0 & 0 & 26 \\
\hline BIOLOGY \& BIOCHEMISTRY & 0 & 46 & 22 & 0 & 0 & 0 & 0 & 0 & 4 & 0 & 2 & 8 & 82 \\
\hline CHEMISTRY & 3 & 7 & 67 & 0 & 40 & 6 & 0 & 0 & 1 & 0 & 0 & 4 & 128 \\
\hline CLINICAL MEDICINE & 0 & 36 & 2 & 0 & 2 & 0 & 0 & 0 & 60 & 0 & 0 & 1 & 101 \\
\hline COMPUTER SCIENCE & 0 & 1 & 0 & 0 & 0 & 0 & 0 & 23 & 2 & 0 & 0 & 0 & 26 \\
\hline ECONOMICS \& BUSINESS & 0 & 0 & 0 & 42 & 1 & 0 & 0 & 0 & 0 & 0 & 0 & 0 & 43 \\
\hline ENGINEERING & 0 & 3 & 1 & 0 & 0 & 3 & 0 & 11 & 0 & 0 & 0 & 5 & 23 \\
\hline ENVIRONMENT/ECOLOGY & 25 & 10 & 6 & 10 & 29 & 22 & 0 & 3 & 0 & 0 & 38 & 63 & 206 \\
\hline GEOSCIENCES & 49 & 2 & 31 & 7 & 100 & 225 & 0 & 30 & 0 & 37 & 108 & 10 & 599 \\
\hline IMMUNOLOGY & 0 & 25 & 0 & 0 & 0 & 0 & 0 & 0 & 12 & 0 & 0 & 0 & 37 \\
\hline MATERIALS SCIENCE & 0 & 4 & 6 & 0 & 5 & 0 & 0 & 0 & 0 & 0 & 0 & 0 & 15 \\
\hline MATHEMATICS & 0 & 1 & 0 & 0 & 1 & 0 & 0 & 13 & 0 & 0 & 0 & 0 & 15 \\
\hline MICROBIOLOGY & 0 & 20 & 1 & 0 & 0 & 1 & 0 & 0 & 4 & 0 & 1 & 0 & 27 \\
\hline MOLECULAR BIOLOGY \& GENETICS & 0 & 44 & 1 & 0 & 2 & 0 & 0 & 0 & 79 & 0 & 0 & 3 & 129 \\
\hline MULTI-DISCIPLINARY & 4 & 46 & 1 & 0 & 3 & 9 & 0 & 5 & 17 & 5 & 9 & 9 & 108 \\
\hline NEUROSCIENCE \& BEHAVIOR & 0 & 2 & 1 & 1 & 0 & 0 & 0 & 0 & 3 & 0 & 0 & 0 & 7 \\
\hline PHARMACOLOGY \& TOXICOLOGY & 0 & 19 & 21 & 1 & 0 & 1 & 0 & 0 & 7 & 0 & 0 & 0 & 49 \\
\hline PHYSICS & 0 & 4 & 12 & 0 & 0 & 8 & 0 & 1 & 0 & 0 & 0 & 0 & 25 \\
\hline PLANT \& ANIMAL SCIENCE & 3 & 34 & 0 & 10 & 8 & 3 & 0 & 4 & 2 & 0 & 8 & 104 & 176 \\
\hline PSYCHIATRY/PSYCHOLOGY & 0 & 0 & 0 & 4 & 0 & 0 & 0 & 0 & 0 & 0 & 0 & 0 & 4 \\
\hline SOCIAL SCIENCES/GENERAL & 1 & 6 & 0 & 15 & 4 & 0 & 1 & 0 & 1 & 0 & 5 & 4 & 37 \\
\hline SPACE SCIENCE & 0 & 1 & 0 & 0 & 0 & 0 & 0 & 0 & 0 & 0 & 0 & 0 & 1 \\
\hline Total & 85 & 332 & 172 & 90 & 195 & 281 & 1 & 90 & 194 & 42 & 171 & 211 & 1.864 \\
\hline
\end{tabular}

aus dem Fachbereich. Außerdem ist keine einzelne Zeitschriftenkategorie erkennbar, die sich gegenüber den anderen deutlich abhebt.

Was die Verbindung zwischen Disziplin, aus der die Autorinnen und Autoren stammen, und Kategorie der Zeitschrift angeht, sind in Tabelle 5 hohe Werte in der Regel dort zu finden, wo Disziplin und Kategorie der Zeitschrift im Wesentlichen das gleiche Forschungsgebiet beschreiben (z.B. Ökologie - Environment / Ecology, Geophysik/Geowissenschaften - Geosciences). Dies kann ein Artefakt der groben Zuordnung zu thematischen Kategorien sowohl der Zeitschrift als auch der Autoren und Autorinnen sein. Allerdings zeigt dies auch, dass über die Publikationsorgane noch kein wirklicher überfachlicher Transfer von wissenschaftlichen Ergebnissen stattfindet, obwohl die Autorschaft interdisziplinär angelegt sein kann. Es scheint, dass trotz interdisziplinärer Kooperation Schwerpunkte gebildet werden, um die disziplinären Gepflogenheiten $\mathrm{zu}$ befriedigen und die Veröffentlichung sicherzustellen.
Tabelle 6 verzeichnet alle Zeitschriften, in denen mindestens zehn Artikel erschienen sind, und ordnet diese noch einmal ihren Journal-Kategorien zu. Von den 17 angegebenen Fachzeitschriften sind etwa die Hälfte der Kategorie Geosciences zugeordnet, was bisherige Ergebnisse (vor allem, dass die Geowissenschaften in verschiedener Hinsicht ein zentrales Gebiet im Exzellenzcluster bilden) stützt. Mit den Fachzeitschriften PloS ONE, Nature und Science sind auch multidisziplinäre Fachzeitschriften darunter.

Die multidisziplinären Zeitschriften, die per definitionem eine große thematische Bandbreite abdecken (Solomon, Carley, \& Porter, 2016), werden im Cluster am häufigsten von den Disziplinen Biologie und Medizin bedient, gefolgt von Ozeanographie und Ökologie. Dies spiegelt zum einen das Interesse der Zeitschriften an diesen Themen wider und deckt sich zum anderen mit dem generellen Impact von Themen aus diesen Disziplinen. Ein Großteil der 100 am häufigsten zitierten Artikel aller Zeiten stammt ebenfalls aus der Biologie (genauer: Verfahren für biologi- 
sche Studien; Van Noorden, Maher, \& Nuzzo, 2014). Interessant ist, dass 17 - und damit die zweithäufigsten - Zuweisungen in multidisziplinären Journalen (s. Tab. 5) aus einem der kleinsten Fachbereiche im Cluster, nämlich der Medizin, stammen.

An Tabelle 6 kann auch abgelesen werden, wie repräsentativ diese Auswahl an Fachzeitschriften für die Gesamtmenge (s. Tab. 1) ist. Von den insgesamt 632 verschiedenen Fachzeitschriften beinhalten die angegebenen 17 je mindestens zehn Artikel. In der Summe sind 346 (von insgesamt 1.380) Artikeln in diesen 17 Fachzeitschriften erschienen. Beachtlich ist also, dass jeder vierte Artikel (25,07 \%) aus dem Exzellenzcluster in knapp 3 Prozent der Fachzeitschriften erschienen ist. Für das Cluster zeigt sich, dass eine kleine Menge an Zeitschriften einen beachtlichen Anteil der Cluster-Publikationen abgedeckt hat.

Tabelle 6: Fachzeitschriften mit mindestens 10 Artikeln aus dem Cluster.

\begin{tabular}{|c|c|c|c|}
\hline $\begin{array}{l}\text { Anzahl } \\
\text { Artikel }\end{array}$ & Fachzeitschrift & ESI-Kategorie & $\begin{array}{r}\text { Impact Factor } \\
(2014)\end{array}$ \\
\hline 48 & $\begin{array}{l}\text { Geochimica et } \\
\text { Cosmochimica Acta }\end{array}$ & GEOSCIENCES & 4,330 \\
\hline 37 & Biogeosciences (BG) & $\begin{array}{l}\text { ENVIRONMENT/ } \\
\text { ECOLOGY }\end{array}$ & 3,976 \\
\hline 27 & $\begin{array}{l}\text { Geophysical Research } \\
\text { Letters }\end{array}$ & GEOSCIENCES & 4,185 \\
\hline 24 & Marine Geology & GEOSCIENCES & 2,710 \\
\hline 23 & $\begin{array}{l}\text { Earth and Planetary } \\
\text { Science Letters }\end{array}$ & GEOSCIENCES & 4,730 \\
\hline 22 & PLoS ONE & MULTIDISCIPLINARY & 3,232 \\
\hline 21 & $\begin{array}{l}\text { Journal of Geophysical } \\
\text { Research - Oceans }\end{array}$ & GEOSCIENCES & $\mathrm{N} / \mathrm{A}$ \\
\hline 21 & $\begin{array}{l}\text { Marine Ecology } \\
\text { Progress Series }\end{array}$ & $\begin{array}{l}\text { ENVIRON } \\
\text { MENT/ECOLOGY }\end{array}$ & 2,618 \\
\hline 18 & $\begin{array}{l}\text { Geochemistry Geophy- } \\
\text { sics Geosystems }\end{array}$ & GEOSCIENCES & 2,923 \\
\hline 17 & Nature & MULTIDISCIPLINARY & 39,327 \\
\hline 17 & Nature Geoscience & GEOSCIENCES & 11,539 \\
\hline 16 & Inorganic Chemistry & CHEMISTRY & 4,760 \\
\hline 13 & Nature Genetics & $\begin{array}{l}\text { MOLECULAR BIOLO- } \\
\text { GY \& GENETICS }\end{array}$ & 28,223 \\
\hline 12 & Marine Biology & $\begin{array}{l}\text { PLANT \& ANIMAL } \\
\text { SCIENCE }\end{array}$ & 2,390 \\
\hline 10 & Ecological Economics & $\begin{array}{l}\text { ECONOMICS \& } \\
\text { BUSINESS }\end{array}$ & 2,719 \\
\hline 10 & Geology & GEOSCIENCES & 4,882 \\
\hline 10 & Science & MULTIDISCIPLINARY & 33,383 \\
\hline
\end{tabular}

\section{Zusammenfassung und Diskussion}

Durch bibliometrische Methoden, i.e. Ko-Autorenanalyse und Boundary Crossing, wurde Evidenz für die interdisziplinäre Zusammenarbeit im Exzellenzcluster The Future Ocean gefunden. Das Cluster eignet sich für eine Bestandsaufnahme, da es explizit die interdisziplinäre Auseinandersetzung mit ozean-relevanten Themen in seinen Fokus gerückt hat. Die Forschenden, und damit nach unserer Operationalisierung auch die Publikationen, des Clusters stammen zum größten Teil aus der Geophysik, Ökologie und der Geowissenschaften. Ein kleinerer Anteil der Cluster-Mitglieder kommt aus den Geisteswissenschaften, der Medizin und Jura.

Die Ko-Autorenanalyse ergab, dass innerhalb des Clusters eine enge Vernetzung zwischen den Fachbereichen besteht, es lassen sich nur wenige isolierte Autoren und Autorinnen feststellen. Es kristallisieren sich verschiedene Communities mit disziplinären Schwerpunkten heraus, aber es sind auch interdisziplinäre Communities vorhanden, die verschiedene Fachbereiche kombinieren und die für solche Forschungssituationen notwendige „,combination of knowledge“ (Abramo, D’Angelo, \& Costa, 2012) gewährleisten. Die größte Interdisziplinarität lässt sich bei Communities feststellen, bei denen Forschende aus den Geisteswissenschaften beteiligt sind. Auf Publikationsebene ist Interdisziplinarität am häufigsten in Ko-Autorschaften zwischen Biogeochemie, Ozeanografie, Ökologie und Geophysik zu finden. Das verdeutlicht, dass im Cluster eher mit thematisch nahverwandten Fachbereichen publiziert wird, was auch schon von Ortega und Antell (2006) gezeigt wurde.

Eine besondere Rolle bei der interdisziplinären $\mathrm{Zu}$ sammenarbeit kommt zentralen Autoren und Autorinnen $\mathrm{zu}$, die eine hohe Betweenness Centrality aufweisen. Im Cluster sind es häufig solche Publizierende, die auch einen hohen Interdisziplinariätsgrad haben, damit wichtige Schlüsselfiguren im interdisziplinären Austausch und bei der Vermittlung von Informationen und Kontakten sind. Ohne diese Knoten im Netzwerk würde das Cluster an Interdisziplinarität verlieren.

Die Publikationen des Clusters sind in allen Kategorien der Thomson ISI Essential Science Indicators erschienen, was die thematische Bandbreite sowie den großen Adressatenkreis reflektiert. Die meisten Artikel stammen aus der Kategorie Geoscience, die Biologie publiziert in den meisten Kategorien. Die Medizin ist, trotz des geringen Anteils an Clustermitgliedern, stark in den multidisziplinären Zeitschriften vertreten. Allgemein lässt sich für das 
Cluster feststellen, dass Boundary Crossing stattfindet, aber dennoch der größte Anteil der Publikationen in solchen Disziplinen erscheint, die mit der Heimat-Disziplin der Forschenden korrespondieren.

Eine deskriptive Bestandsaufnahme ist vor allem von strategischem Wert für die Management-Ebene von interdisziplinären Forschungssituationen, z. B. in Bezug auf die gezielte Zielgruppenansprache und Distribution von Forschungsergebnissen. Vor allem die Analyse der zentralen Clustermitglieder, die Träger und Trägerinnen von Interdisziplinarität, wenn man will, ist wichtig für Steuerung und Entscheidungsprozesse. Soll die Interdisziplinarität gesteigert werden, sollten Maßnahmen eingesetzt werden, die die Entwicklung von zentralen Personen fördert. Andererseits stellt ein Wegfall von zentralen Personen ein Risiko für die Interdisziplinarität dar, das durch flankierende Maßnahmen abgedämpft werden sollte. Die Analyse der Publikationsorte ist von operativem Interesse, bspw. für Bibliotheken, die das Cluster unterstützen. Wie gezeigt wurde, können sie sich nicht nur auf die Literaturbeschaffung zu den Heimatdisziplinen der Forschenden begrenzen, sondern sollten auch die Orte der Rezeption einbeziehen (Ortega \& Antell, 2006).

In dieser Studie wurde bewusst auf die wettbewerbliche Analyse der Future Ocean-Publikationen verzichtet. Eine Zitationsanalyse wäre aber dennoch interessant, vor allem eine Referenzanalyse, um die Wissensbasis des Clusters aufzuzeigen und auch hier Anzeichen von Interdisziplinarität aufzuspüren. Die Studie hat für das Boundary Crossing zudem nur die disziplinäre Zuordnung von Zeitschriftenartikeln betrachtet. Damit sind Disziplinen, die andere Publikationsformate, wie Konferenzen oder Monographien/Sammelbände, bevorzugen, systematisch in der Auswertung benachteiligt worden. Eine Ausweitung der Analyse auf diese Publikationstypen sollte für eine vollständige Bestandsaufnahme durchgeführt werden. Gleichsam könnte eine vollständigere Beschreibung des disziplinären Werdegangs der Forschenden aus dem Cluster weitere Hinweise auf Interdisziplinarität liefern (Ortega \& Antell, 2006). Die Zuordnung der Forschenden zu Institutionen/Arbeitsgruppen und damit zu Disziplinen verschleiert die Tatsache, dass fachfremde Autoren und Autorinnen in der Disziplin tätig sein können (z. B., wenn eine Mathematikerin im Bereich Biologie angestellt ist). Dadurch und durch den in der Methodik begründeten Ausschluss von Publikationen durch Einzelautorschaft unterschätzen unsere Ergebnisse zwangsläufig den tatsächlichen Anteil an interdisziplinären Ko-Autorschaften, die mit den Mitgliedern aus Future Ocean bestehen.

Unsere Ergebnisse können zudem einen Ansatz zur Kategorisierung der Disziplinen liefern. Die mengenmäßig ungleiche Verteilung von Forschenden und Publikationen in den verschiedenen Disziplinen hat jedoch eine grobe Kategorisierung erfordert, um überhaupt Häufungen und strukturelle Eigenheiten feststellen zu können. Laut Parthey (2011) ist für den Erfolg interdisziplinärer Forschung nicht die multidisziplinäre Zusammensetzung der Forschergruppe verantwortlich (obwohl das sicherlich als förderlich angenommen werden kann), sondern die Bereitschaft der einzelnen Forschenden, das „disziplinär fehlende Wissen zur Problembearbeitung [einzuholen] und die daraus resultierende Suche nach Methodentransfer aus anderen Spezialgebieten [durchzuführen] “ (S. 25). Wie bei den meisten bibliometrischen Studien können unsere quantitativen Indikatoren zur Häufigkeit und Korrelation von Interdisziplinarität jedoch keine Aussagen über die Kausalität machen. Hier öffnet sich eine Möglichkeit zu vertiefenden Arbeiten: Eine Inhaltsanalyse könnte die notwendigen Aufschlüsse über das Stattfinden von Interdisziplinarität geben und z. B. zeigen, welche Fragestellungen und Methoden auf welche Weise miteinander verschmolzen sind oder ob gar keine Synthese im Hinblick auf Interdisziplinarität stattgefunden hat.

\section{Literatur}

Abramo, G., D’Angelo, C. A., \& Costa, F. (2012). Identifying Interdisciplinarity Through the Disciplinary Classification of Coauthors of Scientific Publications. Journal of the American Society for Information Science and Technology, 63(11), 2206-2222.

Allmendinger, J. (2015). Quests for Interdisciplinarity: A Challenge for the ERA and HORIZON 2020. Policy Brief by the Research, Innovation, and Science Policy Experts (RISE). URL: https://ec.euro pa.eu/research/innovation-union/pdf/expert-groups/rise/all mendinger-interdisciplinarity.pdf [4.8.2017].

Ball, R., \& Tunger, D. (2005). Bibliometrische Analysen-Daten, Fakten und Methoden-Grundwissen Bibliometrie für Wissenschaftler, Wissenschaftsmanager, Forschungseinrichtungen und Hochschulen (Vol. 12). Forschungszentrum Jülich.

Blondel, V. D., Guillaume, J. L., Lambiotte, R., \& Lefebvre, E. (2008). Fast Unfolding of Communities in Large Networks. Journal of Statistical Mechanics: Theory and Experiment, 2008(10), P10008.

Braun, T., \& Schubert, A. (2003). A Quantitative View on the Coming of Age of Interdisciplinarity in the Sciences 1980-1999. Scientometrics, 58(1), 183-189.

Brown, R. R., Deletic, A., \& Wong, T. H. F. (2015). How to Catalyse Collaboration. Nature, 525, 315-317.

Fischer, K. (2011). Interdisziplinarität im Spannungsfeld zwischen Forschung, Lehre und Anwendungsfeldern. In K. Fischer, H. Laitko, \& H. Parthey (Hrsg.), Interdisziplinarität und Institutionalisierung der Wissenschaft. Wissenschaftsforschung Jahrbuch 2010 (pp. 33-58). Berlin: Wissenschaftlicher Verlag.

Güdler, J. (2013). Fachübergreifende Begutachtung: Strukturwirkung und Fördererfolg Eine Exploration auf Basis von Neuanträgen in 
der DFG-Einzelförderung (2005 bis 2010). URL: http://www.dfg de/download/pdf/dfg_im_profil/zahlen_fakten/statistik/ber icht_fachuebergreifende_begutachtung.pdf [4.8.2017].

Havemann, F., \& Scharnhorst, A. (2010). Bibliometrische Netzwerke. Handbuch Netzwerkforschung, 799-823.

Larivière, V., \& Gingras, Y (2014). Measuring Interdisciplinarity. In B. Cronin \& C. Sugimoto (Eds.), Beyond Bibliometrics: Harnessing Multidimensional Indicators of Scholarly Impact (pp. 187-200). Cambridge MA: MIT Press.

Leahey, E., Beckman, C. M, Stanko, T. L. (2017). Prominent but Less Productive. The Impact of Interdisciplinarity on Scientists' Research. Administrative Science Quarterly, 62(1), 105-139. DOI: $10.1177 / 0001839216665364$.

Ledford, H. (2015). Team Science. Nature, 525(7569), 308-311.

Littke, R. (2009). Publikationsverhalten in den Geowissenschaften. In Alexander von Humboldt-Stiftung (Ed.)., Publikationsverhalten in unterschiedlichen wissenschaftlichen Disziplinen. Beiträge zur Beurteilung von Forschungsleistungen (pp.102-103). URL: https://www.humboldt-foundation.de/pls/ web/docs/F13905/12_disk_papier_publikationsverhalten2_ kompr.pdf [4.8.2017].

Newman, M. E. J. (2001). Scientific Collaboration Networks I. Network Construction and Fundamental Results. Physical Review E, 64(1), 016131.

Noth, J., Rose, A (2009). In Alexander von Humboldt-Stiftung (Ed.)., Publikationsverhalten in unterschiedlichen wissenschaftlichen Disziplinen. Beiträge zur Beurteilung von Forschungsleistungen (pp.110-113). URL: https://www.humboldt-foundation.de/pls/w eb/docs/F13905/12_disk_papier_publikationsverhalten2_ kompr.pdf [4.8.2017].

Ohly, H. P. (2003). Bibliometric Mapping of Selected Research Fields. In Bibliometric Analysis in Science and Research Applications, Benefits and Limitations, Proceedings of the 2nd Conference of the Central Library, Jülich, Germany (pp. 53-62). URL: http:// juser.fz-juelich.de/record/34921/files/Bibliometric_Analysis. pdf [4.8.2017].

Ortega, L., \& Antell, K. (2006). Tracking Cross-Disciplinary Information Use by Author affiliation: Demonstration of a Method. College \& Research Libraries, 67(5), 446-462. DOI: 10.5860/ crl.67.5.446.

Parthey, H. (1995). Bibliometrische Profile der Kaiser-Wilhelm-Gesellschaft zur Förderung der Wissenschaften (1923-1943). (Veröffentlichungen aus dem Archiv zur Geschichte der Max-PlanckGesellschaft, Band 7). Berlin: Archiv zu Geschichte der MaxPlanck-Gesellschaft.

Parthey, H. (2011). Institutionalisierung disziplinärer und interdisziplinäre Forschungssituationen. In K. Fischer, H. Laitko, \& H. Parthey (Hrsg.), Interdisziplinarität und Institutionalisierung der Wissenschaft. Wissenschaftsforschung Jahrbuch 2010 (pp. 9-35). Berlin: Wissenschaftlicher Verlag.

Pierce, S. J. (1999). Boundary Crossing in Research Literature as a Means of Interdisciplinary Information Transfer. Journal of the Association for Information Science and Technology, 50(3), 271-279.

Porter, A., \& Rafols, I. (2009). Is Science Becoming More Interdisciplinary? Measuring and mapping six research fields over time. Scientometrics, 81(3), 719-745.

Rylance, R. (2015). Global Funders to Focus on Interdisciplinarity. Nature, 525(7569), 313-315.
Schummer, J. (2004). Multidisciplinarity, Interdisciplinarity, and Patterns of Research Collaboration in Nanoscience and Nanotechnology. Scientometrics, 59(3), 425-465.

Solomon, G. E. A., Carley, S., \& Porter, A. L. (2016). How Multidisciplinary Are the Multidisciplinary Journals Science and Nature? PLOS ONE 11(4): e0152637. DOI: 10.1371/journal.pone.0152637.

Umstätter, W. (1999). Bibliothekswissenschaft als Teil der Wissenschaftswissenschaft - unter dem Aspekt der Interdisziplinarität. In W. Umstätter \& K.-F. Wessel, Interdisziplinarität - Herausforderung an die Wissenschaftlerinnen und Wissenschaftler, Berliner Studien zur Wissenschaftsphilosophie \& Humanontogenetik (pp. 146-160). Bielefeld: Kleine Verlag.

Van Noorden, R. (2015). Interdisciplinary Research by the Numbers: An Analysis Reveals the Extent and Impact of Research that Bridges Disciplines. Nature, 525(7569), 306-308.

Van Noorden, R., Maher, B., \& Nuzzo, R. (2014). The Top 100 Papers. Nature, 514(7524), 550.

Wasserman, S., \& Faust, K. (1994). Social Network Analysis. Methods and Applications. Cambridge: Cambridge University Press.

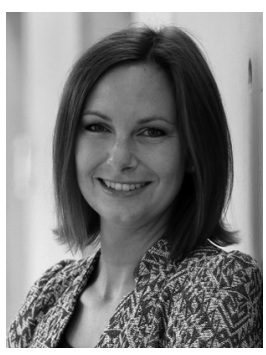

Prof. Dr. Isabella Peters

ZBW - Leibniz-Informationszentrum Wirtschaft \& Christian-Albrechts-

Universität zu Kiel

Düsternbrooker Weg 120

24105 Kiel

i.peters@zbw.eu

Isabella Peters ist Professorin für Web Science am ZBW LeibnizInformationszentrum Wirtschaft und der Christian-Albrechts-Universität zu Kiel. In ihrer Forschung befasst sie sich mit nutzergenerierten Inhalten und wissenschaftlicher Kommunikation, Altmetrics, Science 2.0 und Open Science.

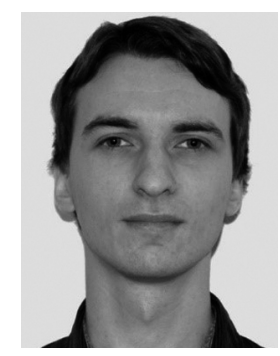

\section{Leon Ladewig}

Technische Universität München Institut für Informatik Boltzmannstraße 3 85748 Garching bei München leon.ladewig@in.tum.de

Leon Ladewig ist Doktorand am Institut für Informatik der Technischen Universität München. Er arbeitet an der Analyse und dem Entwurf von Algorithmen für Optimierungsprobleme. 